\title{
Transport cross sections based on a screened interaction potential: Comparison of classical and quantum-mechanical results
}

\author{
R. Vincent, ${ }^{1}$ J. I. Juaristi, ${ }^{2,3}$ and I. Nagy ${ }^{4,1}$ \\ ${ }^{1}$ Donostia International Physics Center DIPC, P. Manuel de Lardizabal 4, 20018 San Sebastián, Spain \\ ${ }^{2}$ Departamento de Física de Materiales, Facultad de Químicas UPV/EHU, Apartado 1072, 20080 San Sebastián, Spain \\ ${ }^{3}$ Unidad de Física de Materiales, Centro Mixto CSIC-UPV/EHU, P. Manuel de Lardizabal 3, 20018 San Sebastián, Spain \\ ${ }^{4}$ Department of Theoretical Physics, Institute of Physics, Technical University of Budapest, H-1521 Budapest, Hungary
}

(Received 27 January 2005; published 21 June 2005)

\begin{abstract}
Standard classical and quantum-mechanical methods are used to characterize the momentum-transfer cross section needed in energy-loss calculations and simulations for heavy, swift charges moving in an electron gas. By applying a well-known, finite-range screened Coulombic potential energy to model the two-body collision, the quantitative applicability range of the classical cross section is investigated as a function of charge $(Z)$, screening length $(R)$, and scattering relative velocity $(v)$. The a posteriori condition $(Z / R) / v^{2}<1$, as an upper bound for heavy charges, is deduced for this applicability range from the comparative study performed.
\end{abstract}

DOI: 10.1103/PhysRevA.71.062902

PACS number(s): $34.50 . \mathrm{Bw}$

\section{INTRODUCTION}

The use of moving external charges as probes of static and dynamical properties of matter dates back to the earliest days of modern physics. Particularly, the phenomenon of stopping of a target has, especially for heavy intruders, been one of the most important source of information on these coupled properties. The analysis of the phenomena is in a continual progress on both sides, experimental and theoretical, of physical understanding.

In theoretical modeling, the target is usually considered either as an ensemble of independent atomic constituents or as a degenerate homogeneous electron gas. The kinetic description of stopping for these models rests on the cumulative assumption, and the emphasis is on the special role of electron binding and electrostatic shielding, respectively. The common root of inelastic energy loss-namely, the problem of collision between two charges-is of primary importance for these applied models of condensed matter.

The bare interaction between charges is the Coulomb force for which the classical and quantum-mechanical methods give the same, charge-sign and Planck-constantindependent, differential cross section in the threedimensional (3D) case. The important transport cross section, a weighted angle average of the differential one, is divergent for bare Coulomb interactions in 3D.

It is, therefore, necessary to take into account certain screening effects of static or dynamical character [1], the influence of which may be essentially different in classical and quantum descriptions. In addition to the standard concepts for screening, an interesting and quantum-mechanicsbased idea is mentioned at this point. A special regularization for integrated (total and transport) Coulomb cross sections was suggested in Ref. [2], performing a nonasymptotic calculation in which the detector-scattering-center distance appears explicitly as a cutoff in the scattered Coulomb wave.

In his famous study on stopping [1] Bohr deduced, for a bare Coulomb field, the $\left(2 Z e^{2} / \hbar v\right) \gg 1$ constraint as a necessary and sufficient condition for the justification of the clas- sical, orbital consideration of relative motion. The condition $(2 Z / v)>1$ (we use atomic units $e^{2}=\hbar=m_{e}=1$ unless otherwise is stated) is applied in recent (numerical) attempts for heavy ions to justify classical mechanics for transport crosssection calculation with screened interactions [3,4].

Motivated by this condition and comparing with the classical Bohr theory [5] of stopping of harmonically bound electrons, it was demonstrated that the binary-scattering theory could give, with a suitable screening $[3,4]$, equivalent results. On the other hand, as was already pointed out by Bohr, for a screened field the convenient classical variable $\xi=\left[(2 Z / R) / v^{2}\right]$ should also play [1] an important role in a more detailed justification, due to the predominance of largeangle deflections in the transport cross section.

Of course, it is well known that if one makes $\hbar \rightarrow 0$ in any formula of quantum theory, one obtains the corresponding formula in classical theory [6]. As a relevant illustration of this fact, we mention here the exact quantum-mechanical expression $\sigma_{t r}(2 D, v)=\left(2 \pi Z e^{2} / v^{2}\right) \tanh \left[\left(\pi Z e^{2}\right) /(\hbar v)\right]$, obtained for the transport cross section (length) in a $2 \mathrm{D}$ Coulomb field $[7,8]$. In the traditional (3D) terminology for stopping, the $\tanh \left[\left(\pi Z e^{2}\right) /(\hbar v)\right]$ codifies a 2D form for the Bloch correction, which gives the difference between classical and quantum-mechanical treatments in stopping power. Unfortunately, for standard screened Coulomb fields in 3D we have not an exact, analytical result for the transport cross section, from which an expression at the $\hbar \rightarrow 0$ classical limit should follow.

On the other hand, even an exact solution obtained in classical mechanics for the important cross section is not necessarily decisive for an arbitrary combination of parameters (charge, screening length, relative velocity), since it is based on the idea of orbits and impact parameter. Clearly, one can get justification for the applicability range of classical mechanics in stopping description only a posteriori.

As this Introduction shows, the present study is devoted to the understanding at a fundamental level. Thus, apart from selected motivations for the relevant parameter space $(Z, R, v)$, practical applications are not considered. The next 
section, Sec. II contains the theoretical methods and the obtained results. In the last section, Sec. III, a short summary is given.

\section{THEORIES AND RESULTS}

The main goal of the present study, as a natural and logical attempt, is to compare numerically exact quantum results with a classical, analytical expression for the transport cross section using the same screened potential in the Schrödinger and deflection-angle equations:

$$
V(r)=-\frac{Z}{r}\left(1-\frac{r}{R}\right),
$$

with the $V(r)=0$ constraint, for $r \geqslant R$. This form for a screened potential was used in the partial-wave scattering description of electron-atom interactions at the beginning of applied quantum mechanics; for further historical details, see Refs. $[9,10]$.

More recently, motivated by the charge-sign effect in stopping, the above potential with $Z= \pm 1$ (proton and antiproton $[11,12]$ ) and $Z= \pm 2$ has been used [13] to the impactparameter-based description of the transport cross section. In this classical $(c l)$ limit one obtains [11-13] the following:

$$
\sigma_{t r}^{c l}(v)=\pi R^{2} \frac{2}{(A-1)^{2}}[A \ln A-(A-1)]
$$

to which an abbreviation $A=\left[\left(R v^{2} / Z\right)-1\right]^{2}$ is introduced. This analytical expression is based on the simple and informative connection [11] between the deflection angle $(\theta)$ and the impact parameter $(b)$ :

$$
\tan ^{2}\left(\frac{\theta}{2}\right)=\left(\frac{Z}{b v^{2}}\right)^{2} \frac{1-(b / R)^{2}}{\left[1-Z /\left(R v^{2}\right)\right]^{2}} .
$$

We stress the point that the role of screening, with respect to the $R=\infty$ Rutherford form, is twofold. Due to the nominator of Eq. (3) one gets finite cross sections, while via the denominator a charge-sign-dependent, kinetic-energy shift [as Eq. (1) already suggests] becomes apparent and definit.

The expression for the angle in Eq. (3) shows that in the $\left[Z /\left(R v^{2}\right)\right] \rightarrow 1$ (i.e., $\left.A \rightarrow 0\right)$ limit one gets $\Theta \rightarrow \pi$ for the deflection angle at all $0 \leqslant b<R$ values of $b$. This peculiar behavior heralds that the classical parameter $(\xi / 2)=Z /\left(R v^{2}\right)$, introduced by Bohr [1], plays indeed a very important role. This parameter is known [14] now in the field of stopping as the Barkas [15] parameter.

The energy shift results in the $\sigma_{t r}^{c l}(Z, v) \geqslant \sigma_{t r}^{c l}(-Z, v)$ inequality, since the transferred energy $[\Delta E(b)]$ is given, via the $v^{2}(1-\cos \theta)$ expression, by

$$
\Delta E(b)=2 v^{2} \frac{R^{2}-b^{2}}{R^{2}-b^{2}+A b^{2}} .
$$

A similar charge-sign effect, at least for $\left[Z /\left(R v^{2}\right)\right]<1$ [see Eq. (5)], is expected also in the wave-mechanical (Schrödinger) description; the Hamiltonian consists of the same kinetic and potential energy terms.

Notice that in the $\xi \rightarrow 0$ limit the logarithm of Eq. (2) tends to a Bohr-type logarithm $\ln \left(v^{2} R /|Z|\right)$ deduced before quantum mechanics [5] for the classical oscillator model of an atom. Precisely, it is this behavior which governed the development of a binary theory $[3,4]$ based on a numerical solution of the classical scattering problem with a Yukawatype screened potential. In this attempt the parameter $R$ is associated with the electron binding (dynamical character) in the adiabatic limit.

The quantum-mechanical description, for spherical potentials, is based on an expansion for the outgoing wave in terms of angular momentum eigenstates. The radial Schrödinger (with $k \equiv v$, in a.u.) equation for $R_{l}(k, r)$,

$$
\frac{1}{r^{2}} \frac{d}{d r}\left(r^{2} \frac{d R_{l}(k, r)}{d r}\right)+\left[k^{2}-\frac{l(l+1)}{r^{2}}-2 V(r)\right] R_{l}(k, r)=0,
$$

was solved numerically with the standard (regular) boundary condition $R_{l}(k, r \rightarrow 0) \sim r^{l}$, and the scattering phase shifts are obtained from the $R_{l}(k, r \rightarrow \infty) \sim[1 /(k r)] \sin [k r-l \pi / 2$ $\left.+\delta_{l}(k)\right]$ asymptotic form.

A linear scaling of variables, $k \rightarrow \alpha k, r \rightarrow r / \alpha$ (and, of course $R \rightarrow R / \alpha$ ), and $Z \rightarrow \alpha Z$, in the radial equation will not change the phase shift values. Therefore, based on the exact [10] expression:

$$
\sigma_{t r}(k)=\frac{4 \pi}{k^{2}} \sum_{l=0}^{\infty}(l+1) \sin ^{2}\left[\delta_{l}(k)-\delta_{l+1}(k)\right],
$$

in which $\delta_{l}(k)$ are the scattering phase shifts, one has $\sigma_{t r}(\alpha k)=\left(1 / \alpha^{2}\right) \sigma_{t r}(k)$, as in the classical equation (2) where $A$ is not changed under this scaling.

In addition, the above-mentioned kinetic-energy shift is also clearly visible from the equation for $R_{l}(k, r)$ with $\mathrm{Eq}$. (1). In fact, the short-range part of the radial equation corresponds, in our case, to an energy-shifted Coulomb problem which is regularized via the finite $R$.

The values of the input variables $(Z, R, v)$ depend, of course, on concrete physics in both the classical and quantum-mechanical descriptions. In our comparative study of the applicability of classical mechanics in stopping calculation for heavy ions moving with moderately high velocities, we use the $|Z| \in[1,40], v \in[1.5,5]$, and $R \in[1,3]$ sets. High (positive) $Z$ values and relative velocities higher than typical Fermi velocities (at about $r_{s}=1.5$ and $r_{s}=2$ density parameters) of metals are evidently important in various experiments and associated simulations [16].

For very high velocities the velocity-dependent $[12,17]$ Friedel sum of phase shifts, as a constraint for the scattering amplitude, may have a straightforward application with a dynamical $(R \sim v)$ output. In this perturbative limit, where the Lippmann-Schwinger radial equations are solved by plane-wave-based kernels, the first-order Born $(B)$ approximation with our potential gives

$$
\sigma_{t r}^{B}(k)=\frac{4 \pi}{k^{4}} Z^{2} \int_{0}^{2 k R} d x \frac{1}{x}\left(1-\frac{\sin x}{x}\right)^{2} .
$$

For $(k R) \gg 1$ the leading form with logarithmic accuracy is quite simple: $\sigma_{t r}^{B}(k)=(4 \pi)\left(Z / v^{2}\right)^{2} \ln (2 k R)$. This is similar to 
the mentioned [2] expression based on Coulomb-wave regularization, since the incoming wave is only perturbatively modified. Notice that we shall use, in our figures, the mathematically complete $\sigma_{t r}^{B}(k)$ result.

For intermediate velocities and nonperturbative couplings further motivations of reasonable $R$ values are in order. For negative intruders $(Z<0)$ in an electron gas of density $n_{0}$ $=3 /\left(4 \pi r_{s}^{3}\right)$ the Wigner-Seitz picture [18], which rests on a properly constrained depletion hole for repulsive interaction, may fix the $\left(R / r_{s}\right)^{3}=|Z|$ condition on $R(Z)$. For atoms $(Z>0)$ in their ground states, the chemical-radius argument [19], which should be relevant in our one-electron scattering treatment, suggests an about $R \simeq$ unity characteristic quite independently of $Z$. For more detailed tables of the $R(Z)$ parameters, we refer to Ref. [10].

Finally, recent self-consistent screening calculations, performed within density-functional theory for excited embedded atoms, give an $2<R<3$ estimation as a function of the prescribed number of not-populated (hole) bound states [20-23]. A highly excited, screened atom resembles physically to our model in Eq. (1), since the model corresponds, from an electrostatic (Poisson) point of view, to the screening of the point charge $Z$ by a compensating electron distribution whith constant density on a sphere of radius $R$.

Notice that the screening of swift highly charged ions is itself a delicate question. The understanding of associated multiple-electron processes is in its infancy. Conventional concepts for screening, which are based usually on suitable fields for independent (free) electrons, may have limitations on an important portion of the stopping process.

Our detailed results, based on Eqs. (2), (6), and (7), are exhibited in Figs. 1-3. The presentation (but not the calculations) for negative charges is restricted by $|Z| \leqslant 10$, as in a recent atomistic treatment [24]. For compact screening $(R$ is small, Fig. 1) and at low $k$ values we obtain the characteristic, quantum oscillations in the transport cross sections for positive charges. These amplitude oscillations are reduced by growing $k$ and shifted to higher $Z$ values.

The Born approximation, Eq. (7), has a very limited range of applicability. The results of Fig. 1 show that the standard second-order Born approximation, based on plane-wave basis functions up to second order for the scattering amplitude [25], cannot give an improvement for the physically most important positive charges. In fact, the perturbative scaling $\sigma_{t r}(k) \sim\left[a(R, k) Z^{2}+b(R, k) Z^{3}\right]$ would lift the first-order results into the wrong direction at higher velocities, since the $b(R, k)$ coefficient is positive [25]. The next, $Z^{4}$-proportional and negative [14] term could correct this second-order lifting. But the resulting net effect should remain on the wrong direction if the perturbative expansion is a meaningful-i.e., convergent-one. Clearly, in a strong-coupling range the conventional series in terms of $Z$ is not adequate.

The classical result, Eq. (2), shows a remarkable accuracy even for positive ions up to, practically, where a $(Z / v) /(R v)=1$ (i.e., $A=0)$ condition becomes satisfied and quantum oscillations ( $k \equiv v$, in a.u.) set in. We stress that this type of condition is behind the observed [see after Eq. (3)] angle peculiarity in the classical method.

The analysis of the other figure sets, Figs. 2 and 3, supports this important conclusion and adds additional informa-

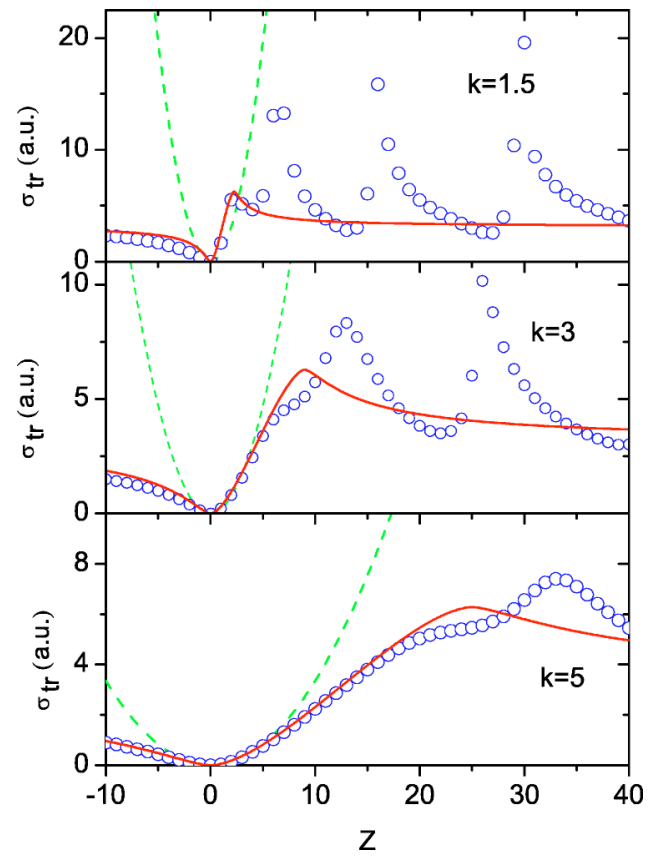

FIG. 1. Transport cross sections as a function of $Z$, at a fixed value of the screening parameter $(R=1)$, and for different relative momenta $(k=1.5,3$, and 5). Open circles refer to quantummechanical calculations and are obtained from Eq. (6). The solid curves are based on Eq. (2), while the dashed ones on the first-order Born approximation given by Eq. (7).

tion to the influence of growing $R$. Clearly, the range for the validity of the first-order Born approximation becomes a gradually (but slowly) wider one. Furthermore, the appearance of quantum oscillations is shifted to higher and higher $Z$ values, showing the combined effect of growing $R$ and $k$, at

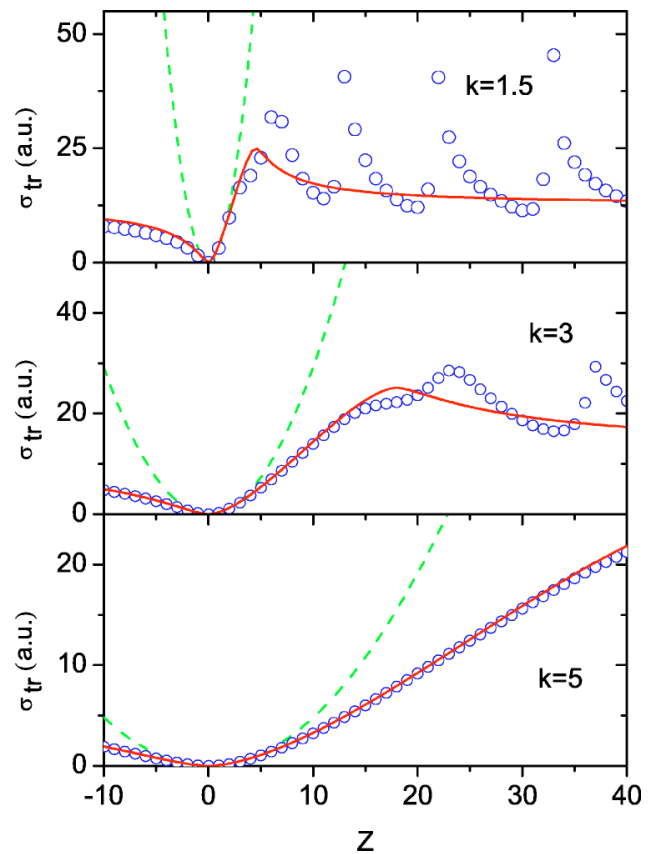

FIG. 2. The same as in Fig. 1 , at the $R=2$ value for the screening parameter. 


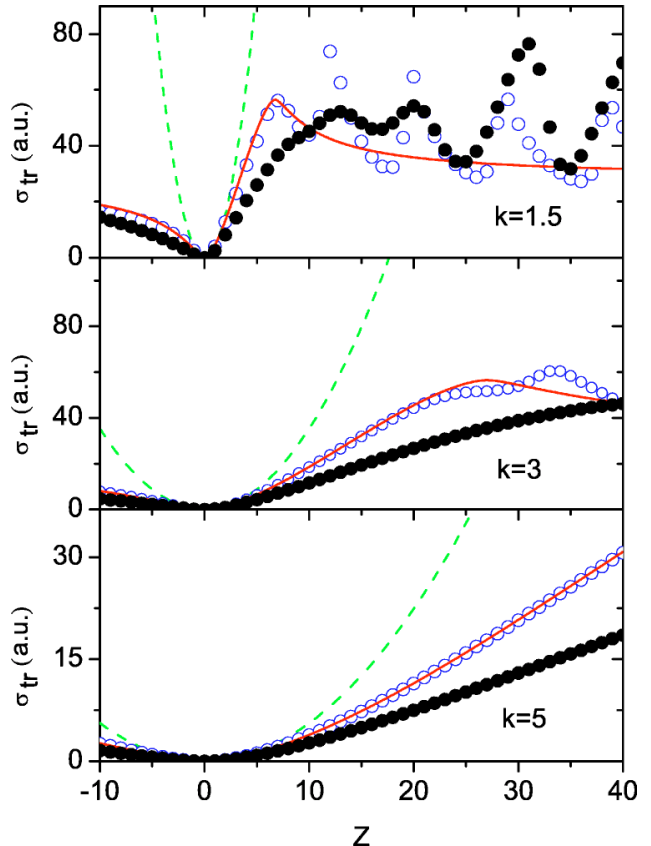

FIG. 3. The same as in Fig. 1, at the $R=3$ value for the screening parameter. Solid circles refer to quantum-mechanical calculations with a rescaled Yukawa potential and are obtained from Eq. (6). (see the text for further details.)

fixed Z. Our deduced scaling argument, together with Figs. $1-3$, could give further orientations in the parameter space.

The observed reduction and shifting in quantum oscillations, obtained here for a finite-range model potential, are in nice agreement with recent quantum calculations performed with a Moliere model (sum of three Yukawa forms) for the interaction [26]. Very recently, a simple rescaling $\lambda=\sqrt{6} / R$ between Eq. (1) and a Yukawa-type $V(r)=-(Z / r) \exp (-\lambda r)$ screened potential has been suggested [27]. This rescaling is based on a perturbatively treated Friedel sum rule and could result in, therefore, quantitatively similar quantum results at higher values of the $R v$ product and for moderate $Z$.

The statement is illustrated in Fig. 3 by solid circles. Physically, the larger screening lenght $[R>(1 / \lambda)]$ in Eq. (1) compensates for the longer spatial range of the Yukawa form [27]. For lower velocities and at high $Z$, the above $Z$ - and $k$-independent scaling becomes only qualitative (due to nonlinearities) and the mapping between potentials needs a separate study.

Other useful information, based on the performed numerics, is related to the interesting $Z$ dependence of the stopping power. There are ranges, always below the quantum oscillations, in which we have an approximately $Z^{n}$ dependence with $n \simeq 1$. This observation is in accordance with earlier numerical ones obtained in Ref. [16], in which the experimental verification is also discussed.
Our analytical expression in Eq. (2), which is based on a classical 3D treatment, provides an accurate representation of this so-called (in a quantum treatment) strong-coupling effect, as the figures show. Notice, parenthetically, that in the classical $(\hbar=0)$ 2D Coulomb case one has [7] a strict $|Z|$ proportionality for any value of $|Z|$.

The observed, remarkable agreement between the quantum and classical results would require a rigorous theoretical analysis of the $\hbar \rightarrow 0$ limit in our 3D quantum case. This analysis may rest on consideration of operators that arise naturally in the study of coherent states [6]. But an investigation of the role of harmonic quantum fluctuations around the classical trajectory is beyond the scope of the present work.

We can state that the classical method is applicable in a remarkably large range of the physical parameters, if they are constrained by $[(Z / v) /(R v)]<1$. This is our main result. It provides an upper bound for $Z$ and shows, in this form, the additional role of the $R v$ product.

\section{SUMMARY}

Motivated by the experimental and theoretical importance of swift, heavy-ion stopping in condensed matter, the capability range of the classical approximation for scattering is investigated in a detailed, comparative study. Based on numerically exact, quantum-mechanical calculations a simple and physically transparent condition $[(Z / v) /(R v)]<1$, as an upper bound in the problem, on this applicability range is deduced. The condition may provide an a posteriori justification of the real validity of recent, numerics-based classical binary theories.

The analytical expression for the transport cross section, together with the established and quite strong condition on its validity, is expected to find applications in various simulation methods. The inelastic energy loss and the nuclear stopping are the key ingredients in such methods devoted, in material science, to the design of target properties. Further works on the stopping-related channels, capture loss, and multielectron processes, could help in constraining the physically relevant, average values of $R$.

\section{ACKNOWLEDGMENTS}

The work of one of us (I.N.) has been supported partly by the OTKA (Grant Nos. T046868 and T049571). He is thankful to Professor A. Galindo for his kind help and to Professor N.R. Arista for useful comments. We acknowledge partial support by the Basque Departamento de Educación, Universidades e Investigación, the University of the Basque Country UPV/EHU (Grant No. 9/UPV 00206.215-13639/2001), and the Spanish MCyT (Grant Nos. BFM2001-0076 and FIS2004-00). 
[1] N. Bohr, Mat. Fys. Medd. K. Dan. Vidensk. Selsk. 18, 1 (1948).

[2] V. G. Baryshevskii, I. D. Feranchuk, and P. B. Kats, Phys. Rev. A 70, 052701 (2004).

[3] P. Sigmund and A. Schinner, Eur. Phys. J. D 12, 425 (2000).

[4] P. Sigmund and A. Schinner, Nucl. Instrum. Methods Phys. Res. B 195, 64 (2002).

[5] N. Bohr, Philos. Mag. 25, 10 (1913).

[6] A. Galindo and P. Pascual, Quantum Mechanics (SpringerVerlag, Berlin, 1991), and references therein.

[7] I. Nagy, Phys. Rev. B 52, 1497 (1995).

[8] E. Zaremba, I. Nagy, and P. M. Echenique, Phys. Rev. B 71, 125323 (2005).

[9] Ph. M. Morse and H. Feshbach, Methods of Theoretical Physics (McGraw-Hill, New York, 1953).

[10] N. F. Mott and H. S. W. Massey, The Theory of Atomic Collision (Clarendon Press, Oxford, 1965).

[11] I. Nagy, Nucl. Instrum. Methods Phys. Res. B 94, 377 (1994).

[12] I. Nagy and B. Apagyi, Adv. Quantum Chem. 46, 267 (2004).

[13] N. R. Arista, P. L. Grande, and A. F. Lifschitz, Phys. Rev. A 70, 042902 (2004).

[14] J. Lindhard, Nucl. Instrum. Methods 132, 1 (1976).

[15] W. H. Barkas, N. J. Dyer, and H. H. Heckman, Phys. Rev. Lett. 11, 26 (1963).

[16] G. Zwicknagel, C. Toepffer, and P. G. Reinhard, Phys. Rep.
309, 117 (1999).

[17] N. R. Arista and A. F. Lifschitz, Adv. Quantum Chem. 45, 47 (2004); A. F. Lifschitz and N. R. Arista, Phys. Rev. A 57, 200 (1998); I. Nagy and A. Bergara, Nucl. Instrum. Methods Phys. Res. B 115, 58 (1996).

[18] I. Nagy, Phys. Rev. B 60, 4404 (1999).

[19] O. J. Heilmann and E. H. Lieb, Phys. Rev. A 52, 3628 (1995).

[20] A. Arnau, P. A. Zeijlmans van Emmichoven, J. I. Juaristi, and E. Zaremba, Nucl. Instrum. Methods Phys. Res. B 100, 279 (1995).

[21] R. Diez Muiño, A. Salin, N. Stolterfoht, A. Arnau, and P. M. Echenique, Phys. Rev. A 57, 1126 (1998).

[22] J. I. Juaristi, A. Arnau, P. M. Echenique, C. Auth, and H. Winter, Phys. Rev. Lett. 82, 1048 (1999).

[23] M. Alducin and J. I. Juaristi, Adv. Quantum Chem. 45, 223 (2004).

[24] P. L. Grande and G. Schiwietz, Adv. Quantum Chem. 45, 7 (2004).

[25] R. G. Newton, Scattering Theory of Waves and Particles (Springer-Verlag, New York, 1983).

[26] N. R. Arista, Nucl. Instrum. Methods Phys. Res. B 195, 91 (2002).

[27] H. B. Nersisyan and A. K. Das, Nucl. Instrum. Methods Phys. Res. B 227, 455 (2005). 